\title{
INTERACTING WITH HUMAN SIMULATIONS: A PROTOTYPE APPLICATION
}

\author{
Ivan Puga-Gonzalez \\ Department of Religion, Philosophy and History \\ University of Agder \\ Universitetsveien 19, 4630 \\ 4604 Kristiansand, NORWAY \\ ivan.puga-gonzalez@uia.no
}

\author{
Saikou Y. Diallo \\ Virginia Modeling Analysis and Simulation Center \\ Old Dominion University \\ 1030 University Blvd. \\ Suffolk, VA, USA \\ sdiallo@odu.edu
}

\begin{abstract}
The process of interacting and executing agent-based simulations of human phenomena is complex and difficult to follow for lay people. This is due to 1) limited access to computing power, 2) difficulty in writing analytics scripts and 3) the size and complexity of the simulation space. Consequently, agent-based simulations are normally used once to answer a limited set of questions instead of exploring all the potential paths that the model offers. Here, we describe an approach for developing user experiences that automate the analytic process and rely on interactive visualizations and life histories of societies and agents. This approach helps the user visualize simulation results, pin-point consequences of different initial conditions on the societies' evolution and agents' behavior, and ultimately allow the user to build a narrative or storytelling to present complex information. We present a prototype application that we implemented in the $\mathrm{R}$ language.
\end{abstract}

Keywords: r-shiny, visual analytics, artificial intelligence, agent-based modeling.

\section{INTRODUCTION}

In the last couple of decades, there has been a rapid growth of agent-based models specifically designed to develop, implement, and test public policies related to complex human social phenomena (Jager and Edmonds 2015; Gilbert et al. 2018). Human social phenomena are often considered complex systems since they involve dozens of factors and thousands or millions of actors that interact and influence each other in complex feedback loops. To represent such degree of complexity, agent-based models have passed from simple models (limited in the number of factors and describing a few patterns of the social phenomena) to models trying to capture all or most of the factors thought to be involved or affecting an specific phenomena. This has resulted in models trying to represent the complexity of social systems at most levels, from the individual to the environmental, institutional and societal level. The development and analysis of such agent-based simulations, however, has mostly remained the same (but see "participatory modelling", Ahrweiler 2010) . First, policy makers meet with model developers to whom they explain in detail the problem and with whom they conceptualize a model. Based on this initial conceptualization, model developers build a model, analyze simulations' results, and validate the model by comparing how well the model reproduces or predicts some empirical data from the system of interest. If the model is considered acceptable, then the results are presented to policy makers who may use this information to create or improve policies. Although this approach seems useful for model developers and policy makers, it, nevertheless, presents some inherent challenges for users of the simulation who were not directly involved in the model conceptualization, building and implementation stages. For instance, without the appropriate 


\section{Puga-Gonzalez and Diallo}

documentation and context, users may find difficult, if not impossible, to understand the factors producing different outcomes in the model. Further, lack of analytical capabilities may preclude users from fully understanding advanced statistical analysis of the factors or processes affecting model results. Furthermore, this approach emphasizes the use of models as predictive devices of system-levels patterns instead of making use of their fully explanatory power (Perry and O'Sullivan 2018).

Agent-based models are built following a bottom-up approach; as such, they offer the possibility of obtaining a deep understanding of how system-level patterns emerge from the interactions of its lower level constituents (i.e. individuals, environment, institutions, etc.) and/or from a series of causal events. Agentbased model thus, can be used as a way of story-telling or narrative on how different macro-level patterns come about. For instance, one may not only explain the initial conditions and parameter values producing a specific pattern at the end of the simulation but also study the series of causal events that derive on a pattern of interest in the simulation. This can greatly facilitate the understanding of phenomena since the narrative aids in the exposition and explanation of a pattern (Millington et al. 2012, Perry and O'Sullivan 2018). We propose an approach for analyzing agent-based models emphasizing their use not only as predictive devices but also as mean to build narratives that may allow users to present complex information in a more friendly and easy-to-grasp way.

Our approach involves the use of interactive visualization and life histories of individuals combined with a quantitative approach. The goal is to 1) give users an overall explanation of the system-level patterns, 2) present them with the opportunity of selecting a pattern of interest and, 3) provide them with the tools that facilitate the construction of a narrative that explains, sequentially, the causal events leading to the emergence of that specific pattern. We realize that doing this already presents several challenges, especially when presented with highly complex models. For example, the large number of factors and inputs parameters included in highly complex models often results in a large parameter space that is difficult to explore when computing power is limited. Further, simulations may generate large quantities of output data (especially when keeping record of each entity and its interactions with other entities, i.e. other agents, institutions, etc.) for which it is difficult to write scripts on how to proper handle and analyze it. Additionally, when documentation or verbal descriptions of the model are separated from simulation results, models may appear as black boxes that only model developers can understand. Given these limitations, it is not surprising that model developers often focus on the analysis at the system level (e.g. societal level) rather than looking at all levels of analysis. Here we show how to overcome all these challenges by following an approach that includes generic functions to handle and analyze data frames in several ways. Then, we show how building an application facilitates the visualization of data and provide users with the adequate tools to understand how user-selected specific patterns come about in the simulations, what were the sequential events leading to this result, which factors are the major predictors of the pattern, and how predictions may change by modifying the value of important factors. Hence, by combining these in-deep exploration and analysis of the model with its documentation, we provide users with the necessary tools to understand patterns at the system level (society level) and individual level, make predictions of the pattern by modifying the values of predictor factors and ultimately build a narrative of the events leading to a pattern. To illustrate our approach, we make use of the individual-based model "Simulation Land" (SimLan). We specifically used this model because its degree of resemblance to Western Societies and human societies in general (see section2) would facilitate the user to empathize with agents in the model and thus evoke a narrative. The rest of the paper is organized as follows. First, we expose our ideas on why building narratives aids in the communication and explanation to lay people of the emergence of social complex phenomena in agent-based models. We then turn to the SimLan model, explain its purpose, architecture and implementation. Next, we show how we analyze and present the results of the SimLan model in a browser application combining interactive visualization of data, life histories of societies and individuals, and predictive models that may ultimately help the user build a narrative. We finalize with an account of the challenges and limitations we faced during the analysis of this model and potential ways of improvement for the future. 


\section{NARRATIVE AND USER EXPERIENCE}

\subsection{Simulation Land Architecture}

SimLan is an agent-based model developed using the AnyLogic multi-method simulation platform, version 8.2 (AnyLogic, 2018). The model represents an extensible artificial society designed with the goal of obtaining a better understanding of the conditions and mechanisms leading to the structural, social, and cultural integration of minorities into large Western societies. SinLam is a spatially explicit 2-dimiensional virtual world representing the city of London and its neighborhoods. The virtual society counts with structural (employment, income, education) and demographic (marriage, reproduction, life expectancy) variables typical of Westerns countries. This society is inhabited by agents that are born, get educated, obtain and lose jobs, get married, reproduce, age, and die. The agents' living location is distributed in different neighborhoods and linked to their job locations. Agents are categorized into two different groups: the majority group, representing the resident population; and the minority group, representing an immigrant population from a single-cultural setting. The model starts with the minority population in place, after the burst of immigration has been completed, bracketing the first decade after the occurrence of the immigration event. Hence, at the beginning of the simulation, the population represents the demographics (age, sex, employment, education, number of children) observed in a western society after the first decade of an immigration event. The model then focuses on the subsequent generations.

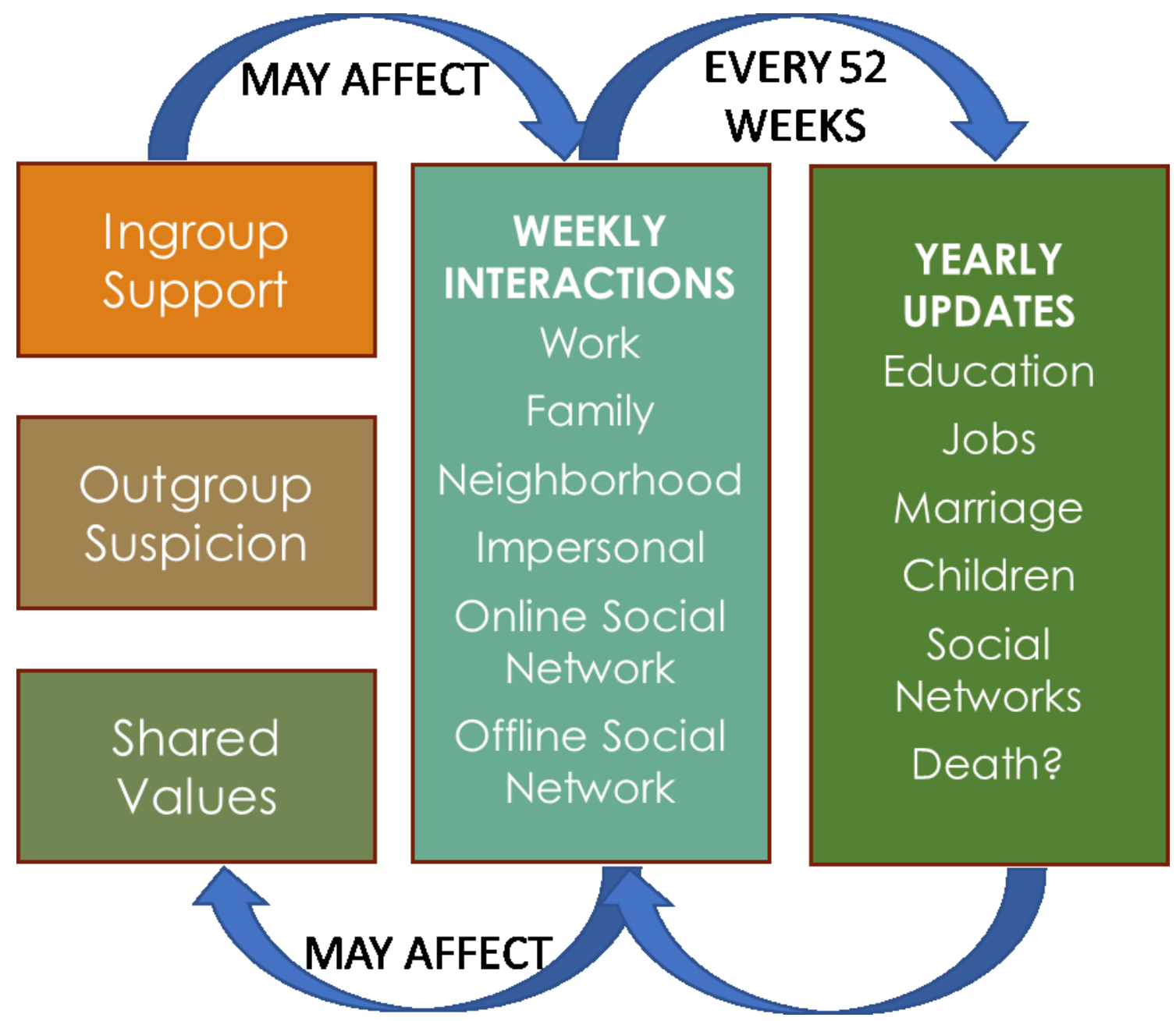

Figure 1: SimLan Concepts and Architecture. 
Agents follow a defined set of actions throughout their lifespan. At initialization the demographic (living location, group, gender, age, education, life expectancy) and societal variables (in group support -IGS-, out group suspicion -OGS-, shared norms -ShNr-) of the agents belonging to the first generation are set. Employment statuses are set based on agents' group and education, and on the percentages of males and females from the majority being employed. Incomes are set based on education and employment status. If agents meet a minimum age, they may get married with a certain probability, and married couples may then have children. In the final step of initialization, agents' online and offline social networks are set. Initialization of agents belonging to generation two and higher is the same except that these agents have their group and neighborhood set the same as that of their parents; current education set to zero, initial occupation status set to student, and initial income set to zero. Further, if they belong to the minority group, their values of $I G S, O G S$, and $S h N r$ are set to the average values of their parents. After initialization, agents enter the recurring sequence of processes of the model. In these recurrent processes, agents above 12 years old have weekly interactions with agents belonging to each up their social networks: neighborhood; online social network, offline social network, impersonal, family, and workplace (if applicable). Agents thus can have up to six different types of interactions every week. During an interaction, an agent randomly picks another from its social network and after the interaction they update their values of $I G S, O G S$ and $S h N r$. After 52 weeks, the agents enter the new year state where a series of updates are performed. Agents age, increase their education, if reaching their maximum level, they move into the working force. Those in the workforce may obtain (if unemployed) or lose (if employed) their jobs. Agents reaching a minimum age may get married, and married couples may have children. Agents' online and offline social networks are updated (new connections are made and some are lost). Finally, agents reaching their life expectancy are declared death and others may experience a sudden death with a low given probability. Agents declared dead are removed from the simulation and the rest return to the weekly interactions state (Figure 1).

\subsection{Agent demographic and integration variables}

Agents are categorized into majority or minority groups depending on their family of origin and they are endowed with variables related to demographic (age, gender, group, education, employment, income, marital status, living location, and life expectancy), and societal integration features (outgroup suspicion, ingroup support, and shared norms). These specific demographic variables were selected since they seem to be the minimal necessary to simulate interesting social dynamics and are useful for policy modeling related to integration of minorities (Niessen 2000). Regarding societal integration, we followed the literature and distinguish three different types of integration: structural, social and cultural integration . Structural integration is characterized by the minority population's acquisition of equal opportunities in education, employment, housing, civil rights and civic participation (Alba and Foner 2015; Hirsch 1987). In the model, this is operationalized by implementing processes representing differences in access to education, to employment, and agents' economic status for the majority and minority population. For instance, each agent receives a specific number of years of education drawn from a statistical distribution mimicking the distribution observed in real minority/majority populations of Western societies. Regarding employment, in the model there are a specific number of job locations which vary in the number of employees and in the number of employees allocate to the minority. Income of agents is based on their years of education. The number of job locations, of employees per job location, and income of agents are drawn from statistical distributions mimicking the distributions observed in Western societies. Social integration refers to the changes in social behavior and attitudes experienced by members of both the minority and majority groups, so they collectively alter the dominant norms of social interaction (Alba and Foner 2015; Hirsch 1987). Social integration was operationalized in two variables, out-group suspicion $(O G S)$ and in-group support (IGS). In-group support represents the desire of agents to display different features of their own culture, such as language, dress, holidays, and religious practices. Out-group suspicion represents the behavior and attitude of agents towards members of the opposite group. Cultural integration is defined as a form of cultural exchange and transformation. The minority population assimilates the norms, values, and cultural capital of the majority population, while the host nation broadens its selfperception to become more inclusive and tolerant of diversity (Alba and Foner 2015; Hirsch 1987). Cultural 
integration was operationalized with a single agent variable, shared norms ( $S h N r)$. This variable represents the extent to which a member of the minority population embraces the general values and culture of the majority population.

On a weekly basis, agents have up to 6 different kind of social interactions. To interact, agents must be at least 12 years old. Interactions occur within the agents' family, neighborhood, online, offline, impersonal, and work networks. Family networks consist of father and mother; neighborhood networks of all agents living in the same neighborhood; online networks of a certain number of agents selected at random from the whole population; impersonal networks of all agents within a radius of spatial proximity to the interacting agent; and work networks of agents working in the same job location. Offline social networks are stochastic, i.e., the probability of an agent being in someone else's network is inversely proportional to the spatial distance between the agents. Online and offline social networks are updated every year, during the update agents may gain and loose connections with other agents. Work networks are updated every time the agent gets, loses, or changes employment. Every week, an agent selects an interaction partner from each of its networks. Interactions result in positive, negative, or neutral experiences which increase, decrease, or leave equal the agent's variables related to social $(I G S, O G S)$ and cultural integration $(S h N r)$-figure 1-. Interactions among the majority produce no change in the integration variables; interactions among the minority or inter-group interactions have a positive or negative impact on the value of the integration variables. The perception of an interaction being negative or positive depends on the current average values of $\mathrm{ShNr}$ and $O G S$ variables in the population.

\section{USE CASE AND RESULTS}

A summary of the procedure followed to generate and analyze the data is show in figure 2. We sampled the parameter space (26 input variables) using latin hypercube sampling (Stein 1987) with the 'lhs' $r$ library (Carnell 2018). As recommended by Prowse et al. (2016), we simulated each parameter combination just once but swept the parameter space in detailed (5,000 different combinations of input parameter values). We acknowledge that we did not confirm whether the parameter sampling was sufficient; for our purposes however, 5000 simulations gave us enough data to work on. From each simulation, we collected data every simulation 'year' at the society and individual level. At the society level we keep track of demographic changes in percentages of: group (majority/minority), gender (male/female), occupation status (employed, unemployed, student), marital status (single/married), etc. Likewise, we keep track of changes in the average societal values of $I G S, O G S$, and $S h N r$. At the individual level, every simulation 'year' we recorded the values of the demographic and integration variables of each agent. To analyze the data collected we used R statistical software version 3.5.1 (Team-R-Core 2015). We ran GLM models and a machine learning method, XGBoost tree, to understand the relationship between the input conditions and the values of the integration variables at the society and individual level. To do so, we first created two generic functions to automatize the analysis of the data. The first generic function used the 'leaps' $\mathrm{R}$ library to perform multimodel inference (Lumley 2017). This function returns the best linear model (based on the adjusted R2) via the function regsubsets(). For each of the integration variables (IGS, OGS, ShNr) and each analysis level (society and individual) we created a GLM model. For models at the society level, we use all of the input parameters $(n=26)$ plus simulation 'year' as predictors; the 'yearly' average of the integration variable was the response variable. At the individual level, we used the same predictors plus the agents' demographic variables, and the agents' average social network (family, neighborhood, online, offline, and work social network) value of the integration variable; as response variable we used the agents' integration variable value. The second generic function used the 'xgboost' R library (Chen et al. 2018). The 'xgboost' R library uses supervised learning, i.e. it infers a function that maps an input (vector of predictors) to an output (response). To do so, the data is first divided into two different sets called 'train' and 'test' data. Next, the supervised learning algorithm analyzes the training data and produces an inferred function. This function is then used to predict the unseen instances (output variables) from the test data (input variables). Our data set was then divided into $80 \%$ of the train phase and $20 \%$ for the test phase. In an optimal scenario, the algorithm will with great accuracy determine the values of the unseen instances in the 'test' data set. In our 
case, the xgboost tree achieved a high accuracy at the society level ( $95 \%)$ but a low one at the individual level $(\sim 20 \%)$. The function inferred by 'xgboost' $\mathrm{R}$ library is based on a set of decision trees. In machine learning, decision trees are used to go from observations (input variable) represented in tree's branches; to measurement of the target item (output variable), represented in tree's leaves. Trees where the response variable takes discrete values are called classification trees, and those in which the response variable takes continuous values are called regression trees. Usually, the 'xgboost' library produces an ensemble of trees, this ensemble sums the predictions of multiple trees together. We should note that 'xgboost' models do not produce significance tests, however they do identify the importance of the predictor variables on the basis of how frequently they appear in all ensemble trees or by calculating the gain in accuracy by including an specific factor in the tree's branches. As for the GLM models, we created a xgboost model for each integration variable and each level of analysis. The predictors and response variables used for the xgboost models were the same as for the GLM (see above).

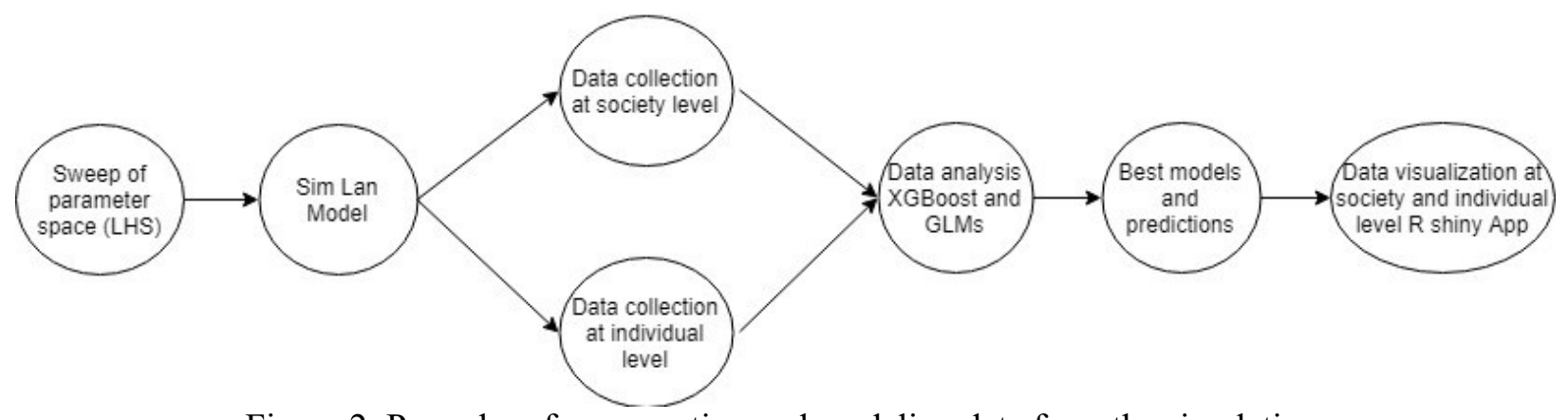

Figure 2: Procedure for generating and modeling data from the simulation.

\subsection{Shiny application}

To explore and visualize the analysis of the data in better detail beyond statistics summaries, we build an $\mathrm{R}$ shiny web application: https://sim-lan-r-shinny-app.shinyapps.io/SimLanApp/. 'Shiny' is a R library that enables users to build interactive web apps from R (Chang et al. 2018). Given its interactive nature, the application then allows users to modify and visualize the analysis of data in different ways. The user thus, it is not restricted to the results presented by the model analyzer but can actively request to analyze and visualize the data in different ways (see below). We used 'shiny' in combination with 'shinydashboard' $r$ library (Chang and Ribeiro 2018) to structure and subset the application in different tabs and subtabs. The application is divided in four main tabs: Home, Analytics, Society, and Individual; each of them with their own subtabs. The home tab is subdivided in three different subtabs: welcome, documentation, and about us. The welcome subtab gives a brief description of the Sim Lan model. The documentation tab, on the other hand, presents the user of the application with in-deep details of the architecture of the model, simulation, and of the agent's variables and interactions. In this way the user has a document that provides $\mathrm{him} / \mathrm{her}$ with all relevant information to understand the architecture, interactions, and dynamics of the model without having to rely on an expert/model developer explanation. The analytics tab allows the user to have a quick glimpse of the initial conditions and final output values of all simulations ran, as well as to visualize any potential correlation among them. The tab is organized in four different plots (Figure 3B). The top two plots are histograms, each of them with a drop-down menu of input/output parameters to select from. For instance, by selecting the "Initial Ingroup Support maj" for histogram 1, the user can visualize the values taken by this parameter in all of the simulations ran. Similarly, by selecting "Final out group Suspicion maj" the user gets an overview of all the values of this specific variable generated by the model in all the simulations ran. The two bottom plots are correlations plots. The left plot is a 2-dimensional plot and the right one a 4-dimensional plot. These two plots allow the user to explore potential correlations between any of the input/output variables presented in the drop-down menus. In summary, the analytics tab allows the user to get a quick exploration of all simulations ran in the model. The histograms allow to explore all initial and final conditions' values of the model and thus rapidly spot if the model has a blind 
spot in the parameter space or whether it generates all possible values (or values of interest) of the target variable at the end of the simulation.

The society tab is divided in two subtabs: exploration and simulation. The exploration subtab allows the user to select a specific simulation run (i.e. a society based on a specific set of final conditions) from all the simulations ran and explore it in detail (Figure 3A). For instance, users could select a society in which cultural integration is high (high average value of shared norms), but social integration low (high average values of in group support and out group suspicion). In this tab, the first and second row of the screen show demographic data such as the number of simulation years, the current population size, number of generations, the percentage of agents belonging to the minority/majority group, male/female gender, etc. The third and fourth row show data related to the integration variables. The histograms show the distribution of the values of each variable hold by agents in the society at a given simulation year. The last plot shows the evolution of the average value of each integration variable in time. The data of the society can be further categorized into that for the majority/minority group, or for a specific group of agents with user-specify attributes. This is done by selecting one of the menu items at the top left, below the 'exploration' title. Most relevant is the possibility of analyzing a specific group of agents selected by the user (Figure 4A). Users can select a group of agents based on specific attributes, such as male agents that are married and employed, and compare the data of this specific group with that of the whole society (Figure 4A-B). Further, at the bottom of the 'specific group' item, the user is presented with GLM models for each of the integration variables using only data from the specific group selected; here a summary statistic table is presented as well as the percentage of variance explained by the model (Figure 4B).

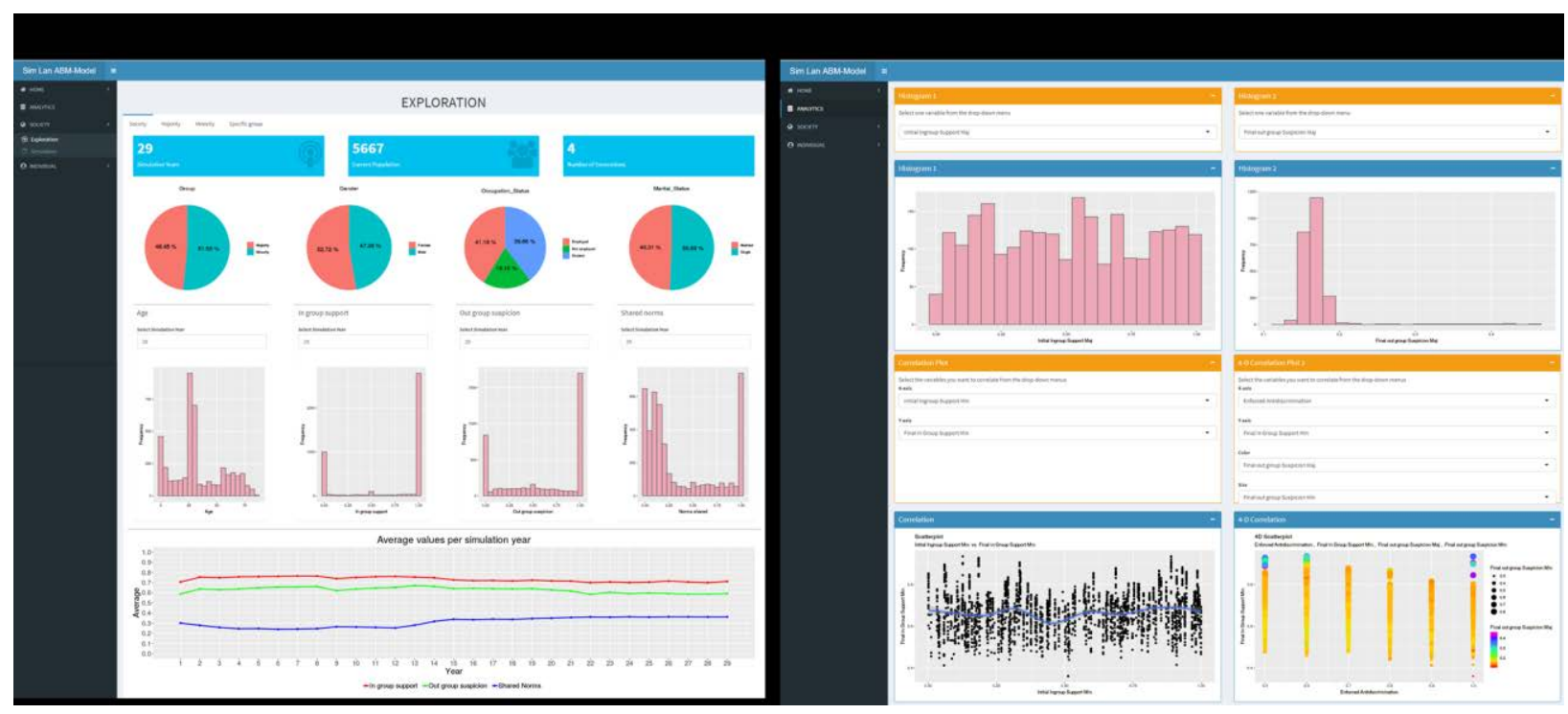

Figure 3: Sample View of the Exploration tab (A) and the analytics tab (B).

The detailed information presented in the exploration tab provides the user with all the necessary information to form a narrative of what may have led a society into a specific direction or value of the integration variables. For instance, plots of the evolution of the average values of the integration variables allow the user to link sudden value changes with specific events in the model. A sudden decrease in the percentage of jobs available for the minority, for example, may lead to a decrease in social (high values of ingroup support and out group suspicion) and cultural integration (low values of shared norms). The user thus is not only able to select a specific society from the pool of all societies simulated but at the same time is presented with all the necessary information to build a narrative of the chain of events that lead a society to its current conditions. In combination with the statistics summaries, this allows the user to get a better understanding of the dynamics governing and steering the value of the integration variables in a society. 
In the simulation subtab, the user is presented with the results of the XGboost tree machine learning analysis at the group level: majority and minority. Here, the user can set the initial conditions of a given society and predict the final values of integration variables. For each integration variable, a different XGBoost tree model was built, each of these models had a predictive accuracy of $\sim 0.95$. Note that not all initial conditions affect the final values of the integration variables, to guide the user regarding which variables have a higher impact on each of the final values of the integration variables, the user can click the button "See importance of parameters" at the bottom of the box. When clicking this button, the user is presented with three plots showing the importance of each of the initial input conditions on each of the integration variables.

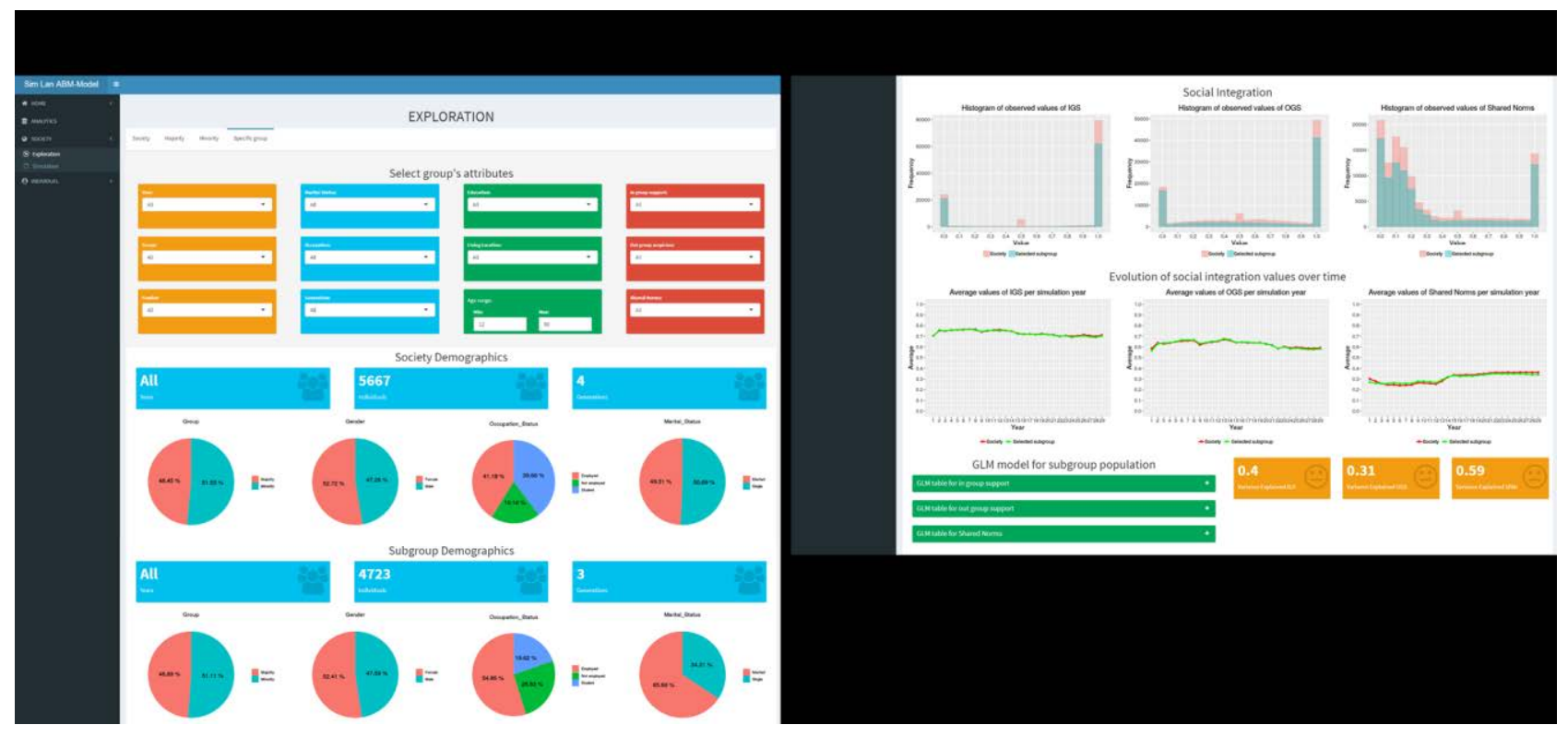

Figure 4: Parameter selection (A) and predictive model result (B).

By building a shiny application, model developers are able to present and visualize model results in a way that is readily accessible for experts and lay people. This may potentially improve not only the way of analyzing a model and spotting problems but also the communication between experts and policy makers. Further, being a web application, people all around the world can have access to it, and given its documentation and interactive nature, there is no need for ad hoc explanations. The user would be able to grasp a good understanding of how and why the simulation generates different societal outcomes by forming its own narrative regarding the causation of the emergent phenomena with little or no guidance from experts. In addition to the analysis at the society level, the user can further zoom in and look into the details of specific individuals in a given society. This is of great importance because most individual-based simulations remain only at the exploration of group-level patterns without going into the details or life histories of specific agents. By zooming into life histories of specific agents, the user can achieve two goals. First, one can assess whether the events leading to specific changes in the agents' variables are potentially relevant and similar to those happening in real human societies, as some kind of face validation of the model. Second, if face validation is met, then users can explore life histories of agents in a similar fashion as that of individuals in human societies. They can thus explore the effect that different live events or changes in policy may produce on specific individuals. Further, policies can be targeted to specific groups of agents or specific places in the model (neighborhoods) and then explored the effects of these policies at the society and individual level. Our approach thus, opens the door to a new way of exploring, analyzing and interpreting individual based models. This bring the potential of better understanding not only to model developers but also to policy makers and lay people, allowing then for a full exploitation of these new tools which may become the pillar of policy experiments in the future. 


\section{LIMITATIONS, CHALLENGES AND FUTURE DIRECTIONS}

The full exploration of the parameter space in the model is one of the main limitations we encountered. In the present work we run $\sim 5,000$ simulations, however, given our parameter space (26 inputs), 5,000 simulations may seem insufficient. We therefore relied on the use of machine learning and generalized linear models to provide simulation results without having to execute more simulations. We acknowledge however, that the accuracy of these models, specially those at the individual level, will only improve when more data becomes available. It is thus of our interest to keep running simulations and add their results to the application so predictions become better. Nevertheless, to do so, we still need relatively large amounts of computational resources given that the simulation of one society for 30 years and with $\sim 10,000$ individuals may take up to one week. Another big challenge is the large amount of data generated at the individual level. Simulations consist of societies with 5,000 and 10,000 individuals, which considering all societies, accounts for over 35 million individuals. Given that we collect individual data every year, the total amount of data already rounds the terabytes size. Hence, we need to explore sampling methods that identify groups of agents that are meaningful to follow (instead of the whole population) over multiple generations. Finally, we acknowledge that in the application, the formation of narratives is limited to the user interpretation of results. The application so far does not create a written narrative. Instead presents all the elements available in the model so the user can build one. Obviously, this may lead to bias given that different users may interpret results in different ways and thus create different narratives. In future studies, we will investigate the possibility of creating written narratives from the elements and events occurring in the model.

\section{CONCLUSIONS}

The idea of creating a statistical equivalent to a computational model and building a user experience to allow people to interface with us gives several advantages. We are able to abstract the inherent complexity of the computational model and provide simulation results without having to execute a simulation. This combined with fine-grain exploration of individuals' and societies' life histories allow us to more thoroughly explore the behavior space of a model and validate it empirically when data is available. A more powerful concept is the reliance on narrative to personalize the content of the user experience so that everyone can access results without having to write scripts of code. Although the present application does not generate any written narrative, it does move one step towards it by providing users with a visualizations and life histories that aid pointing out chain of events leading to specific type of societies or individual situations and thus a narrative.

\section{REFERENCES}

Alba, R., Foner, N. 2015. Strangers No More: Immigration and the Challenges of Integration in North America and Western Europe. Princeton University Press.

Carnell, R. 2018. 1hs: Latin Hypercube Samples.

Chang, W. \& Ribeiro, B. B. 2018. shinydashboard: Create Dashboards with 'Shiny'.

Chang, W., Cheng, J., Allaire, J., Xie, Y. \& McPherson, J. 2018. Shiny: Web Application Framework for $\mathrm{R}$.

Chen, T., He, T., Benesty, M., Khotilovich, V., Tang, Y., Cho, H., Chen, K., Mitchell, R., Cano, I., Zhou, T., Li, M., Xie, J., Lin, M., Geng, Y. \& Li, Y. 2018. Xgboost: Extreme Gradient Boosting.

Gilbert, Nigel, Ahrweiler, Petra, Barbrook-Johnson, Pete, Narasimhan, Kavin Preethi and Wilkinson, Helen (2018) 'Computational Modelling of Public Policy: Reflections on Practice' Journal of Artificial Societies and Social Simulation 21 (1) 14. DOI: 10.18564/jasss.3669

Hirsch. E.D. Jr. (1987) Cultural Literacy: What Every American Needs to Know. Houghton Mifflin. 
Jager, W. \& Edmonds, B. (2015) Policy Making and Modelling in a Complex world. In Janssen, M., Wimmer, M. and Deljoo, A. (eds.) Policy Practice and Digital Science. Springer, pp. 57-74. DOI:10.1007/978-3-319-12784-2_4

Lumley, T. 2017. Leaps: Regression Subset Selection.

Millington, J. D. A., O'Sullivan, D. \& Perry, G. L. W. 2012. Model histories: Narrative explanation in generative simulation modelling. Geoforum; Themed issue: Spatialities of Ageing, 43, 1025-1034. doi: https://doi.org/10.1016/j.geoforum.2012.06.017.

Perry, G. L. W. \& O'Sullivan, D. 2018. Identifying Narrative Descriptions in Agent-Based Models Representing Past Human-Environment Interactions. Journal of Archaeological Method and Theory, 25, 795-817. doi: 10.1007/s10816-017-9355-x.

Prowse, T. A. A., Bradshaw, C. J. A., Delean, S., Cassey, P., Lacy, R. C., Wells, K., Aiello-Lammens, M., Akçakaya, H. R. \& Brook, B. W. 2016. An efficient protocol for the global sensitivity analysis of stochastic ecological models. Ecosphere, 7, e01238. doi: 10.1002/ecs2.1238.

Stein, M. 1987. Large Sample Properties of Simulations Using Latin Hypercube Sampling. Technometrics, 29, 143-151. doi: 10.1080/00401706.1987.10488205.

Team-R-Core. 2015. R: A Language and Environment for Statistical Computing. Vienna, Austria: R Foundation for Statistical Computing.

\section{AUTHOR BIOGRAPHIES}

IVAN PUGA-GONZALEZ is a post-doctoral student at the university of Agder. His research interest are modeling and simulation of complex adaptive systems that are manmade. He holds a $\mathrm{PhD}$ in Theoretical Biology from the University of Groningen. His email address is ivan.puga-gonzalez@uia.no.

SAIKOU Y. DIALLO is a Research Associate Professor at the Virginia Modeling, Analysis, and Simulation Center (VMASC) where he serves as the head of the Simulated Empathy lab. He is also an adjunct Professor of Modeling, Simulation, and Visualization Engineering at Old Dominion University (ODU). He received his MS and $\mathrm{PhD}$ in Modeling and Simulation from ODU. His email address is sdiallo@odu.edu and his web page is http://www.odu.edu/vmasc/research/simulated-empathy.html. 